\title{
Design and Fabrication of Abrasive Jet Machine
}

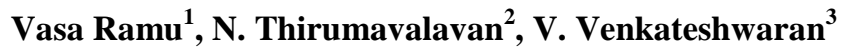 \\ Assistant Professor, Department of Mechanical Engineering, R.M.K College of Engineering and Technology, \\ Puduvoyal, Thiruvallur, India ${ }^{1}$ \\ UG Student, Department of Mechanical Engineering, R.M.K College of Engineering and Technology, Puduvoyal, \\ Thiruvallur, India ${ }^{2,3}$
}

\begin{abstract}
Abrasive Jet Machining (AJM) is the process of material removal from a work piece by the application of a high speed stream of abrasive particles carried in a gas medium from a nozzle. The material removal process is mainly by erosion. The AJM will chiefly be used to cut shapes in hard and brittle materials like glass, ceramics etc.AJM is an effective machining process for processing a variety of Hard and Brittle Material. And has various distinct advantages over the other non-traditional cutting technologies, such as, high machining versatility, minimum stresses on the work piece, high flexibility no thermal distortion, and small cutting forces. It also known as micro-abrasive blasting. It is a mechanical energy based unconventional machining process used to remove unwanted material from a given work piece. The process makes use of an abrasive jet with high velocity, to remove material and provide smooth surface finish to hard metallic work pieces.
\end{abstract}

Keywords: Air compressor, Air filter, Pressure Gauge, Pressure Regulator Vibrator or Mixer, Nozzle, Horizontal and Vertical motion module (for xy motion).

\section{INTRODUCTION}

Abrasive Jet Machining (AJM) is the removal of material from a workpiece by the application of a high speed stream of abrasive particles carried in gas medium from a nozzle. The AJM process differs from conventional sand blasting in that the abrasive is much finer and the process parameters and cutting action are carefully controlled.

The process is used chiefly to cut intricate shapes in hard and brittle materials which are sensitive to heat and have a tendency to chip easily. The process is also used for deburring and cleaning operations. AJM is inherently free from chatter and vibration problems. The cutting action is cool because the carrier gas serves as a coolant.

\section{SCHEMATIC LAYOUT OF ABRASIVE JET MACHINE}

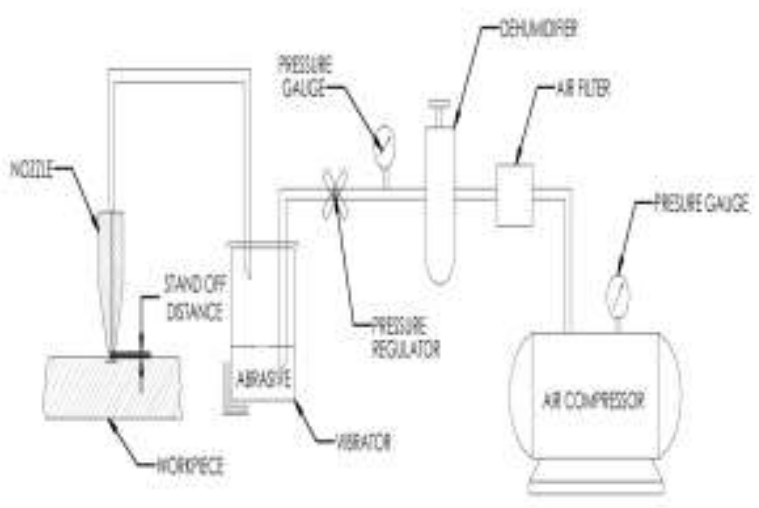

\section{Working Principle}

Dry air or gas is filtered and compressed by passing it through the filter and compressor. A pressure gauge and a flow regulator are used to control the pressure and regulate the flow rate of the compressed air. Compressed air is then passed into the mixing chamber. In the mixing chamber, abrasive powder is fed. A vibrator is used to control the feed of the abrasive powder. The abrasive powder and the compressed air are thoroughly mixed in the chamber. The pressure of this mixture is regulated and sent to nozzle. The nozzle increases the velocity of the mixture at the expense of its pressure. A fine abrasive jet is rendered by the nozzle. This jet is used to remove unwanted material from the workpiece. 


\section{IARJSET

Objective and Aim Of Work:

Abrasive Jet Machining (AJM) is the process of material removal from a work piece by the application of a high speed stream of abrasive particles carried in a gas medium from a nozzle. The major field of application of AJM process is in the machining of essentially brittle materials and heat sensitive materials like glass, quartz, sapphire, semiconductor materials, mica and ceramics. It is also used in cutting slot, thin sections, countering, drilling, deburring, for producing integrate shapes in hard and brittle materials.

1) Ability to cut intricate holes shape in materials of any hardness and brittleness.

2) Ability to cut fragile and heat sensitive material without damage.

3) No change in microstructure as no heat is generated in the process.

4) Low capital cost.

\section{Process Parameters of AJM:}

\section{- $\quad$ Nozzle}

The abrasive particles are directed into work surface at high velocity Through nozzle. Therefore the material of nozzle is subjected to a great Degree of abrasion wear and hence these are made of hard such as tungsten carbide or synthetic sapphire. Tungsten carbide nozzle is used for circular $\mathrm{c} / \mathrm{s}$ in the range of 1 to $3 \mathrm{~mm}$ dia.

\section{- $\quad$ Stand Off Distance}

Standoff distance off nozzle tip distance (NTD) is define as the Distance between the face of the nozzle and the working surface of the work Piece. SOD has been found to have considerable effect on the MRR as well as Accuracy

\section{- $\quad$ Carrier Gas}

Carrier gas to be used in AJM must not flare excessively when discharged from the nozzle into the atmosphere. Further he gas should be nontoxic, cheap, easily available and capable and being dried and cleaned without difficulty. Air, nitrogen and carbon dioxide is generally used as carrier gas. Commercially filled cylinder gases can also be us satisfactorily. Air is widely owing availability and little cost.

- $\quad$ Size Of Abrasive Grain

The rate of metal removal depends upon the size of the abrasive grain. Fine grain are less irregular in shape in hence posses lesser cutting ability. More over finer grains tends to stick . together and check the nozzle . the most Favorable grain size from 10 to 50 microns, coarse grains recommended for Cutting whereas their grains are useful in polishing, debarring etc.

\section{- $\quad$ Velocity Of The Abrasive Jet}

The kinetic energy of the abrasive jet is utilized for metal removal by Erosion. FANNIE \& SHELDON ( ref. ASME code book ) have shown that for Erosion to occurs. The jet must impinge the work surface with a certain Minimum velocity for the erosion of glass by silicon carbide ( grain size 25Micron) the minimum jet velocity has been found to be $150 \mathrm{~m} / \mathrm{sec}$ The jet velocity is the functions of nozzle pressure, nozzle design, abrasive grain size and that mean no. of abrasive nit volume of the carrier gas.

\section{- Work Material}

AJM is recommended for the progressing of the brittle material such as Glass, ceramic ,refractory, semiconductors, cement carbide etc. most of the Ductile material are practically unmachinable by AJM . The rate of metal removal Has been found to depend upon the mohr' s hardness of material to be machined.

\section{- $\quad$ Shape Of Cut}

The accuracy of machining is also dependent upon the shape of cut. It May not be possible to machine component with sharp corners because of stry cutting.

1. $\quad$ Model Of Ajm

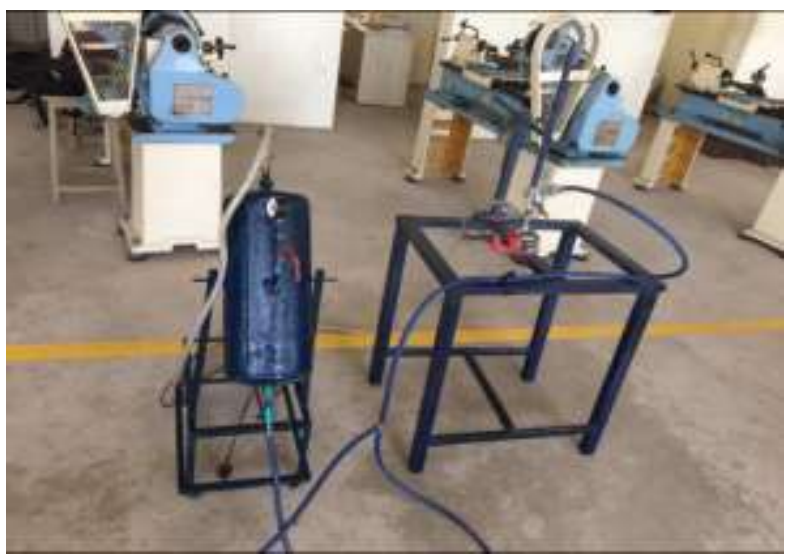


2. Steps To Be Followed In Starting The Machine

- Load abrasive feeding chamber with required grit size of abrasive

- Ensure that all the pipe fitting are air tight and leak proof

- Start the compressor to built up the necessary pressure of about 2 to $8 \mathrm{~kg} / \mathrm{cm}^{2}$

- Fix the nozzle of known diameter to the tapered rod and place the cap properly

- Place the workpiece to be machined in the fixture. Adjust the stand off distance

- Close the dust collection box and cover properly

Applications Of Ajm

1) CLEANING

2) SEMICONDUCTORS

3) CRYSTALLINE MATERIAL

4) STEEL MOULDS

5) DEBURRING

6) MISCELLANEOUS MATELWORKINGAPPLICATON

7) TESTING ABRASION RESISTANT OF VARIOUS MATERIALS

8) MISCELLANEOUS LABORATORY APPLICATION

Merits of Ajm

1) It has ability to cut intricate holes in material of any hardness and brittleness.

2) It is possible to machine fragile, brittle and heat sensitive material without damage.

3) The process can be utilized conventionally in drilling, cutting, deburring etching, polishing and cleaning operations.

4) High surface finish can be achieved.

5) Depth of surface damage is low.

6) The process is characterized by low capital investment and low power consumption.

7) The initial cost of AJM is low.

Demerits Of Ajm

1) Limited capacity owing to low material rate

2) Nozzle wear rate is high.

3) There must be suitable dust collecting system, as the process ends to pollute the environment

Result Discussion

Effect Of Parameters

1) Stand of distance.

2) Abrasive flow rate.

3) Abrasive grit size.

4) Nozzle distance.

Discussion

1) Effect of S.O.D on MRR

2) Effect of flow rate on MRR

3) Effect of size of partical on MRR

Photographs

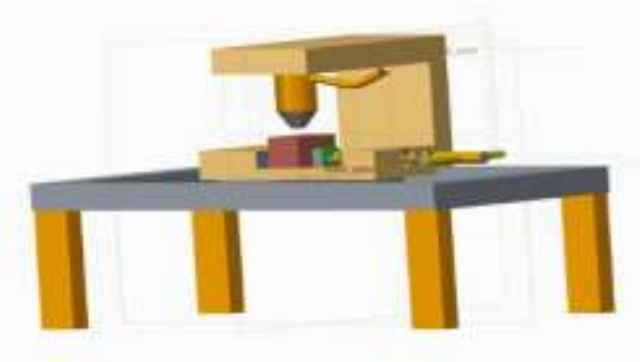

Fig.1.The Abrasive Jet Machine 


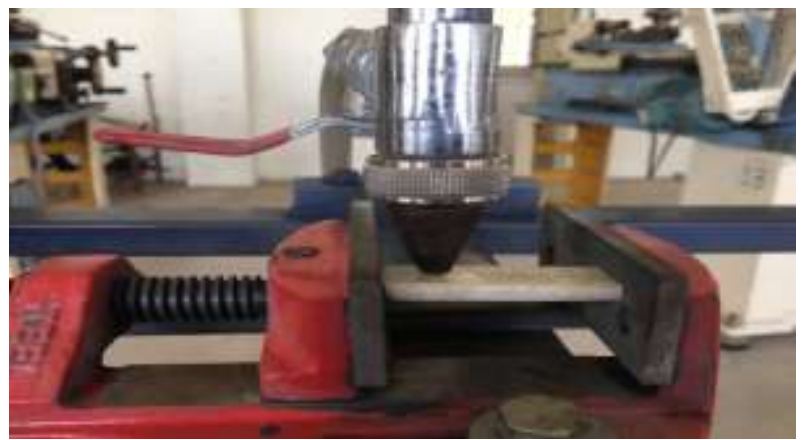

Fig.2.Drill Vice Holding The Workpiece

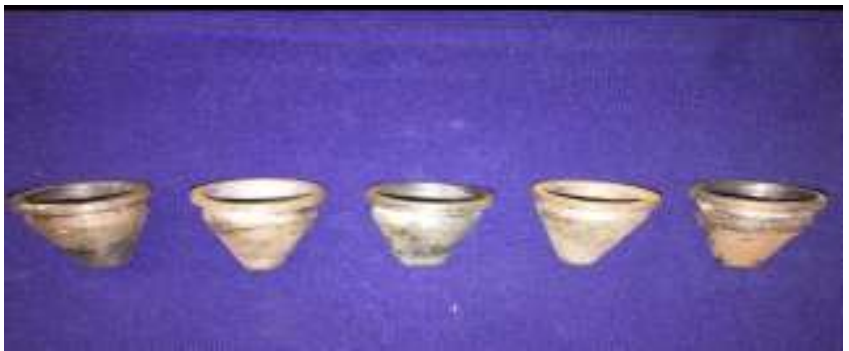

Fig.3.Nozzle Of Different Diameters

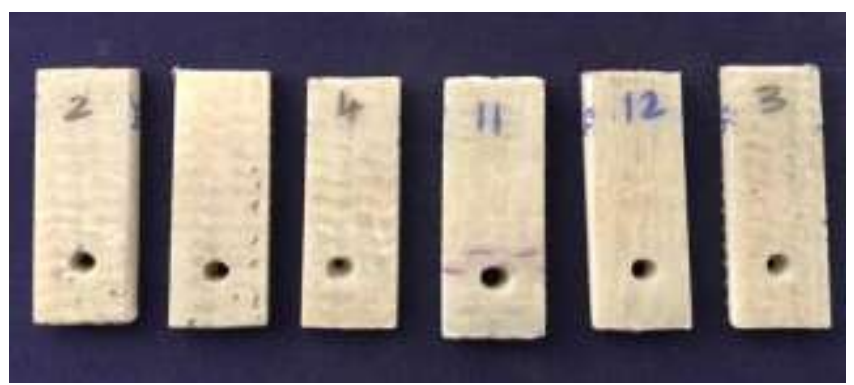

Fig.4.Workpieces After Machining Process

\section{III.CONCLUSION}

Due to low capital and operative cost the ajm is compatible to other processes. In future with slight modifications, ajm will become a important machine tool on shop floor.

There is some scope for future works

- Study of nozzle wear

- Dimensional analysis for calculating theoretical metal removal rate.

- Study of characteristic of abrasive particles and types of abrasive particles.

\section{REFERENCES}

[1] Residual stress and tribological characteristics of ground surface after abrasive jet restricted by grinding wheel Authors: Liu, F., Gong, Y.-D., Shan, Y.-Q., Cai, G.-Q.

[2] Publication: Journal of Northeastern University, Volume 30, Issue 3, Pages 422-425 March 2009.

[3] Simulation and analysis of abrasive jet machining with wheel restriction in grinding Authors: Wang, W.S., Zhu, L.D., Yu, T.B., Yang, J.Y., Tang, $\mathrm{L}$.

[4] Publication: Key Engineering Materials, Volume 389-390, Pages 387-391, 2009

[5] Abrasive waterjet turning-An efficient method to profile and dress grinding wheels Authors: D.A. Axinte, J.P. Stepanian, M.C. Kong, J. McGourlay

[6] Publication: International Journal of Machine Tools and Manufacture, Volume 49, Issues 3-4, March 2009, Pages 351-356 Date: Dec, 2008

[7] Modeling and simulation for material removal in abrasive jet precision finishing with wheel as restraint. Authors: Li, C.H., Ding, Y.C., Lu, B.H.

[8] Publication: Proceedings of the IEEE International Conference on Automation and Logistics, ICAL 2008, Article number 4636666, Pages 2869-2873, 2008

[9] Authors: Ghobeity, A.; Spelt, J. K.; Papini, M. Publication: Journal of Micromechanics and Microengineering, Volume 18, Issue 5, pp. 055014.Publication Date: 01/ 05/2008 